\title{
Entecavir versus tenofovir in patients with chronic hepatitis B: Enemies or partners in the prevention of hepatocellular carcinoma
}

\author{
Sung Won Lee ${ }^{1,2, *}$, Jonggi Choi ${ }^{3, *}$, Seung Up Kim ${ }^{4,5}$, and Young-Suk Lim ${ }^{3}$ \\ ${ }^{1}$ Department of Internal Medicine, College of Medicine, The Catholic University of Korea, Seoul; ${ }^{2}$ The Catholic University Liver Re- \\ search Center, Seoul; ${ }^{3}$ Department of Gastroenterology, Liver Center, Asan Medical Center, University of Ulsan College of Medicine, \\ Seoul; ${ }^{4}$ Department of Internal Medicine, Institute of Gastroenterology, Yonsei University College of Medicine, Seoul; ${ }^{5}$ Yonsei Liver Cen- \\ ter, Severance Hospital, Seoul, Korea
}

Over the past several decades, entecavir (ETV) and tenofovir disoproxil fumarate (TDF) have remained the first-line antiviral agents in several international guidelines. These two antiviral agents have shown similar short to intermediateterm efficacy, including virologic, biochemical, serologic, and histologic responses. However, huge controversies regarding the antiviral efficacy of ETV and TDF in preventing the development of hepatocellular carcinoma (HCC) still exist. In this review, we summarized recent studies that compared the treatment efficacy of ETV and TDF in terms of HCC development. (Clin Mol Hepatol 2021;27:402-412)

Keywords: Entecavir; Tenofovir; Hepatitis B; Carcinoma, Hepatocellular; Efficacy; Outcome

\section{INTRODUCTION}

Chronic hepatitis B (CHB) is known to be the most common chronic viral infection, affecting approximately 350 million people worldwide. ${ }^{1}$ Since the persistent replication with necroinflammation by the hepatitis B virus (HBV) significantly raises the risk of developing compensated cirrhosis and hepatocellular carcinoma
$(\mathrm{HCC}){ }^{2}$ antiviral therapy to suppress HBV replication, which can prevent the progression of liver disease by stabilizing necroinflammation and inducing fibrosis regression, has been the mainstay in the management of patients with CHB. ${ }^{3}$ Several recent studies have proven that oral antiviral agents, particularly entecavir (ETV), reduce the risk of long-term complications such as liver cirrhosis and HCC, ultimately improving survival compared to controls. ${ }^{4}$

\section{Abbreviations:}

AGEs, advanced glycation end products; aHR, adjusted hazard ratio; ALT, alanine aminotransferase; $\mathrm{CHB}$, chronic hepatitis $\mathrm{B} ; \mathrm{Cl}$, confidence interval; ETV, entecavir; $\mathrm{HBV}$, hepatitis B virus; HCC, hepatocellular carcinoma; IPTW, inverse probability of treatment weighting; LT, liver transplantation; NUC, nucleos(t)ide analogue; PSM, propensity score-matched; PY, person year; TDF, tenofovir disoproxil fumarate

*Sung Won Lee and Jonggi Choi equally contributed to this work as cofirst authors.

\section{Corresponding author : Seung Up Kim}

Department of Internal Medicine, Yonsei University College of Medicine, 50-1 Yonsei-ro, Seodaemun-gu, Seoul 03722, Korea

Tel: +82-2-2228-1944, Fax: +82-2-362-6884

E-mail:ksukorea@yuhs.ac

https://orcid.org/0000-0002-9658-8050

\section{Young-Suk Lim}

Department of Gastroenterology, Asan Medical Center, University of Ulsan College of Medicine, 88 Olympic-ro 43-gil, Songpa-gu, Seoul 05505, Korea

Tel: +82-2-3010-5933, Fax: +82-2-485-5782

E-mail:limys@amc.seoul.kr

https://orcid.org/0000-0002-1544-577X 
Sung Won Lee, et al.

Entecavir versus tenofovir for $\mathrm{HCC}$ prevention

Although tenofovir alafenamide and besifovir dipivoxil maleate have been recently available, ${ }^{3,5}$ ETV and tenofovir disoproxil fumarate (TDF), which are potent nucleos(t)ide analogues (NUCs) with a high genetic barrier to resistance, have been the first-line antiviral agents in several international guidelines over the last several decades. ${ }^{3,6,7}$ These two antiviral agents have similar short to intermediate-term clinical efficacy (including virologic, biochemical, serologic, and histologic responses) and similar efficacy for preventing liver disease progression. ${ }^{8,9}$ However, several recent studies have proposed the superiority of TDF over ETV in reducing the risk of HCC development, ${ }^{10}$ whereas other following studies have shown no statistical difference. ${ }^{11,12}$

In this review, we summarized recent studies which compared the treatment efficacy of ETV and TDF in terms of HCC development.

\section{ENTECAVIR AND TENOFOVIR EQUALLY PRE- VENTS HCC}

\section{Hospital-based cohort studies}

\section{Asian studies}

Since Choi et al. ${ }^{10}$ first reported that TDF showed superior effects compared to ETV for the prevention of HCC, more than 10 comparative studies between ETV and TDF with adjusted hazard ratio (aHR) for HCC incidence have been published, as summarized in Tables 1, 2 and Figure 1.

A multicenter retrospective cohort study from Korea analyzed 2,897 patients, and the annual HCC incidence was statistically similar between the ETV and TDF groups in multivariate analysis (aHR, 0.98; 95\% confidence intervals [Cls], 0.75-1.28; $P=0.852$ ), propensity score-matched (PSM) analysis (aHR, 1.02; 95\% Cls, $0.77-1.35 ; P=0.884)$, and inverse probability of treatment weighting (IPTW) analysis (aHR, 1.00; 95\% Cls, 0.77-1.30; $P=0.988$ ) (Table 1). ${ }^{11}$

Another multicenter retrospective cohort study from Korea analyzed 3,022 patients, and found statistically similar incidence rates of HCC between ETV and TDF after PSM in the whole cohort (aHR, 1.08; $95 \% \mathrm{Cls}, 0.52-2.24 ; P=0.842$ ) and in subgroups of patients with chronic hepatitis and liver cirrhosis. In addition, statistically similar incidence rates of all-cause mortality or liver transplantation (LT) between ETV and TDF were observed after PSM in the whole cohort (aHR, 0.98; 95\% Cls, 0.36-2.62; $P=0.961)$ and in patients with chronic hepatitis and liver cirrhosis (Fig. 1). ${ }^{12}$

A multicenter retrospective cohort study from Taiwan analyzed
7,248 patients. The HCC incidence rates of ETV and TDF groups were statistical similar in the whole cohort (aHR, 0.82; 95\% Cls, 0.66-1.02; $P=0.078$ ) and in the PSM cohort (aHR, 0.83; 95\% Cls, $0.65-1.06 ; P=0.129)^{13}$

A single-center retrospective cohort study from Korea analyzed 1,340 patients, and showed that HCC risk was statistically similar between ETV and TDF groups, either by PSM (aHR, 2.06; 95\% Cls, 0.98-4.33; $P=0.058$ ) or IPTW (aHR, 1.30; 95\% Cls, $0.81-$ 2.10; $P=0.276)^{14}$

A single-center retrospective cohort study from Korea analyzed 1,794 patients. Multivariate analysis showed that the risk of HCC and death or LT was statistically similar between ETV and TDF groups (aHR, 0.83; 95\% Cls, 0.52-1.31; $P=0.413$ and $a H R, 0.64$; 95\% Cls, 0.26-1.57; $P=0.325$, respectively) after adjusting for adherence to medication and maintained virologic response. In the 589 PSM patients, the risk of HCC and death or LT was also statistically similar between the two groups (aHR, 0.77; 95\% Cls, $0.46-1.29 ; P=0.319$ and $\mathrm{aHR}, 0.64 ; 95 \% \mathrm{Cls}, 0.30-1.38 ; P=0.257$, respectively). ${ }^{15}$

In a retrospective cohort study by an Asian international consortium which analyzed 5,537 patients, TDF was associated with a lower risk of HCC in the unadjusted analysis. However, in the multivariate analysis, no difference was found between ETV and TDF (aHR, 0.81; 95\% Cls, 0.42-1.56; $P=0.52$ ) after adjustment for age, sex, country, serum albumin, platelet count, alpha fetoprotein, liver cirrhosis, and diabetes mellitus. Furthermore, PSM analysis $(n=1,040)$ found no significant association between antiviral agents and HCC risk in the multivariable-adjusted analysis (aHR, 0.89; 95\% Cls, 0.41-1.92; $P=0.77$ ). ${ }^{16}$

In a multicenter retrospective study from Korea which analyzed 1,560 patients, the incidence of HCC was statistically similar between ETV and TDF groups after multivariate analysis and in the PSM population (aHR, 1.30; 95\% Cls, 0.80-2.02; $P=0.295$ ). ${ }^{17}$

Even before Choi et al. ${ }^{10}$ suggested the superiority of TDF for the prevention of HCC, a single-center retrospective study from Korea had analyzed 582 patients for a median follow-up of 57 months and compared the effects of ETV and TDF. In this study, HCC developed in $6.5 \%$ of the patients, regardless of the type of antiviral agents. ETV and TDF-treated patients showed statistically similar HCC development rates $(P=0.471) .^{18}$

From a slightly different perspective, one Korean multicenter retrospective cohort study compared the effects of ETV and TDF in 726 patients following curative treatments of HCC, such as hepatic resection or radiofrequency ablation. The results showed no association between the type of antiviral agents and HCC recur- 


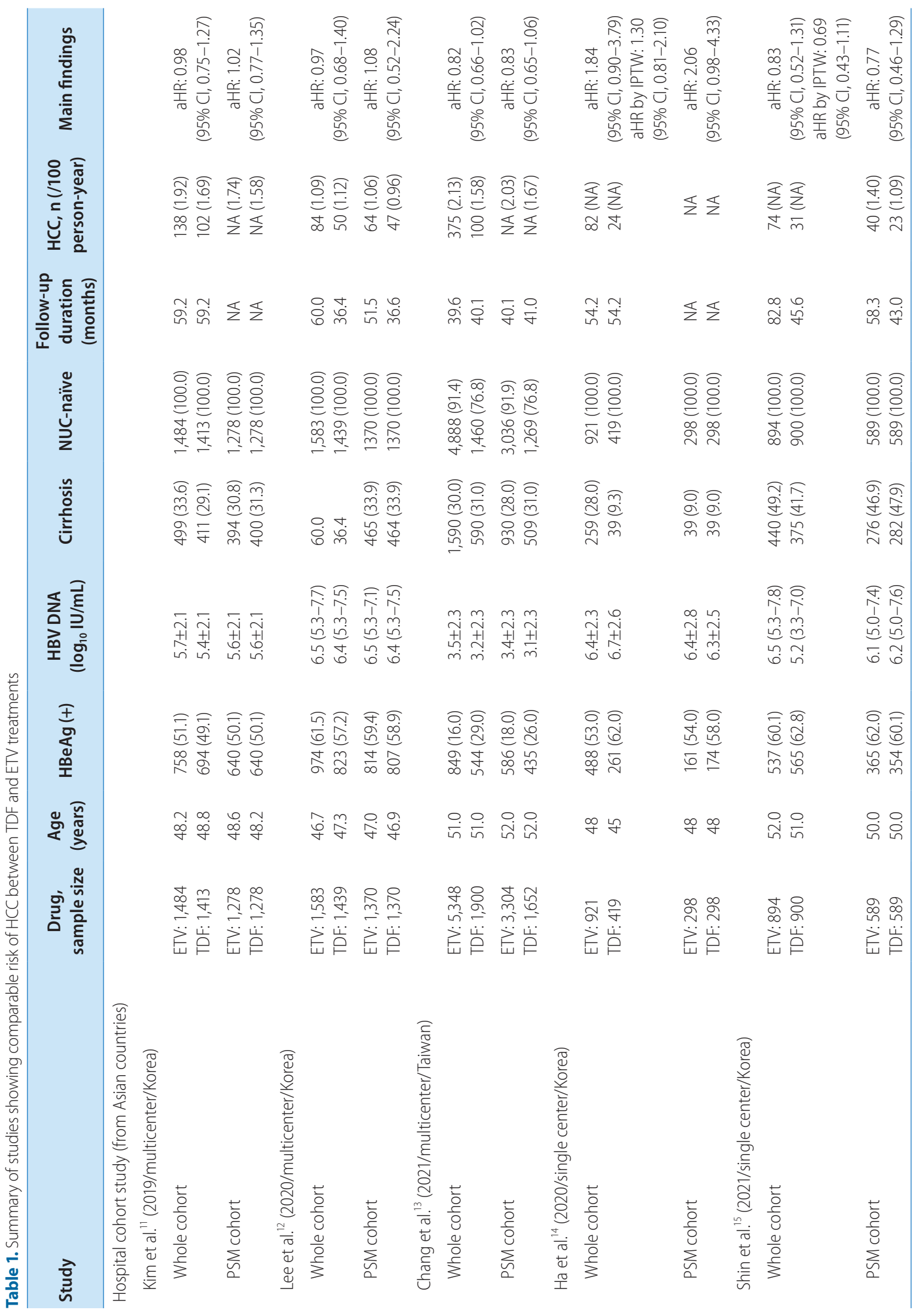




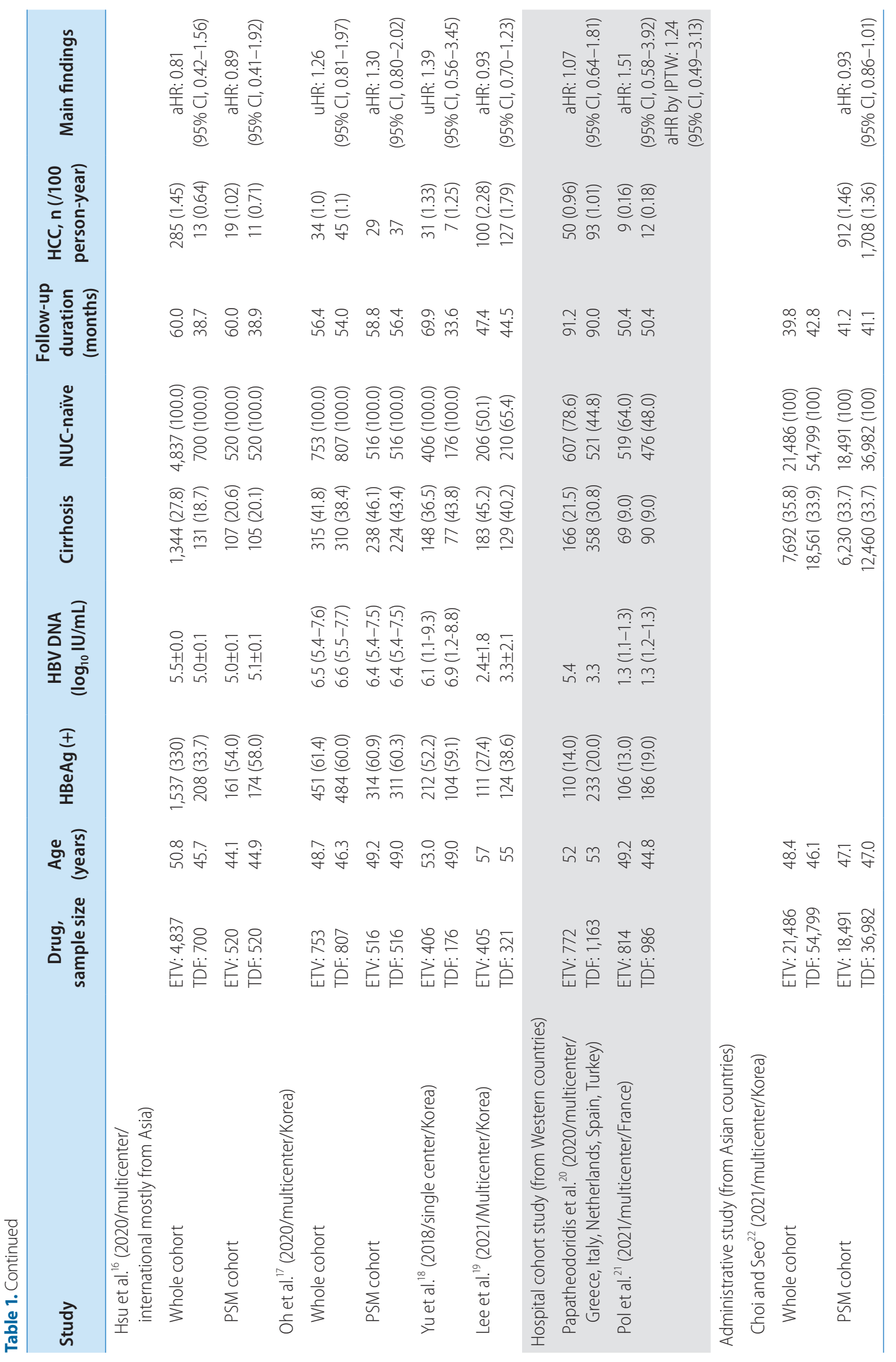




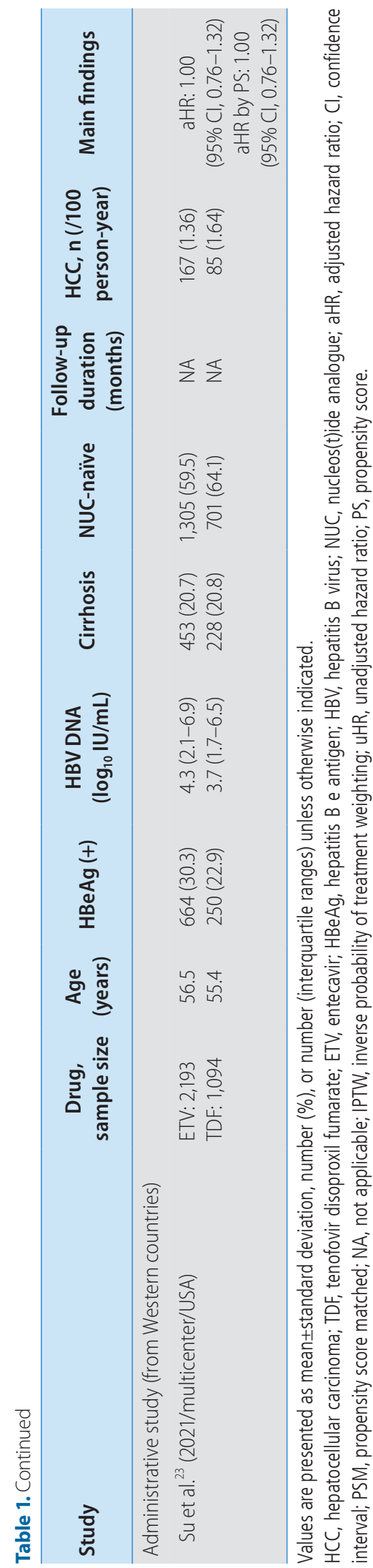

rence (aHR, 0.93; 95\% Cls, 0.70-1.23; $P=0.622$ ) and death (aHR $0.67 ; 95 \% \mathrm{Cls}, 0.36-1.23 ; P=0.193)$ after multivariate analysis. IPTW analysis showed similar results in terms of recurrence (aHR, 1.04; $P=0.963$ ) and death (aHR, 0.80; $P=0.431$ ). In addition, the early ( $<2$ years) and late ( $\geq 2$ years) recurrence risks were statistically similar in the two groups (both $P=0.400$ ), as confirmed by IPTW analysis ( $P=0.502$ and $P=0.377$, respectively). ${ }^{19}$

\section{Western studies}

A multicenter prospective study from Europe that enrolled patients from the PAGE-B cohort analyzed 1,935 Caucasians with CHB, with or without compensated liver cirrhosis, for a median follow-up period of 7.1 years. After multivariate analysis, the hazard ratio of HCC was statistically similar between ETV and TDFtreated patients after adjusting for several HCC risk factors, such as age, sex, smoking, diabetes, alanine aminotransferase (ALT) level, platelet count, hepatitis B e antigen, prior treatment experience, and liver cirrhosis (aHR, 1.07; 95\% Cls, 0.64-1.81; $P=0.791){ }^{20}$

Another multicenter prospective cohort study from France included 1,800 patients for a median follow-up period of 4.2 years. The HCC incidence (aHR, 1.51; 95\% Cls, 0.58-3.92) and all-cause mortality (aHR, 0.60; 95\% Cls, 0.25-1.46) were statistically similar between the ETV and TDF-treated groups. In addition, IPTW analysis showed similar results in HCC occurrence (aHR, 1.24; 95\% Cls, 0.49-3.13) and all-cause mortality (aHR, 0.63; 95\% Cls, $0.28-1.44)^{21}$

\section{Studies using administrative databases}

\begin{abstract}
Asian study
A large-scale retrospective cohort study using nationwide claims data from the Korean Health Insurance Review and Assessment Service enrolled 76,285 patients. ${ }^{22}$ This study analyzed 55,473 treatment-naiive cases where ETV or TDF treatment were started between 2013 and 2017 after matching according to age, sex, comorbidities, hospital type, and index date year for a median follow-up period of 41.2 months. The incidence of HCC was statistically similar between the ETV and TDF groups (aHR, 0.93; $95 \% \mathrm{Cls}, 0.86-1.01 ; P=0.081)$. Interestingly, in the subgroup analysis of patients who had started antiviral therapy during the 2012-2014 period, which was identical to the enrollment period in the landmark study by Choi et al., ${ }^{10}$ the incidence of HCC was higher in the ETV group compared to the TDF group (aHRs, 0.85; 95\% Cls, 0.79-0.91; $P<0.001)$.
\end{abstract}




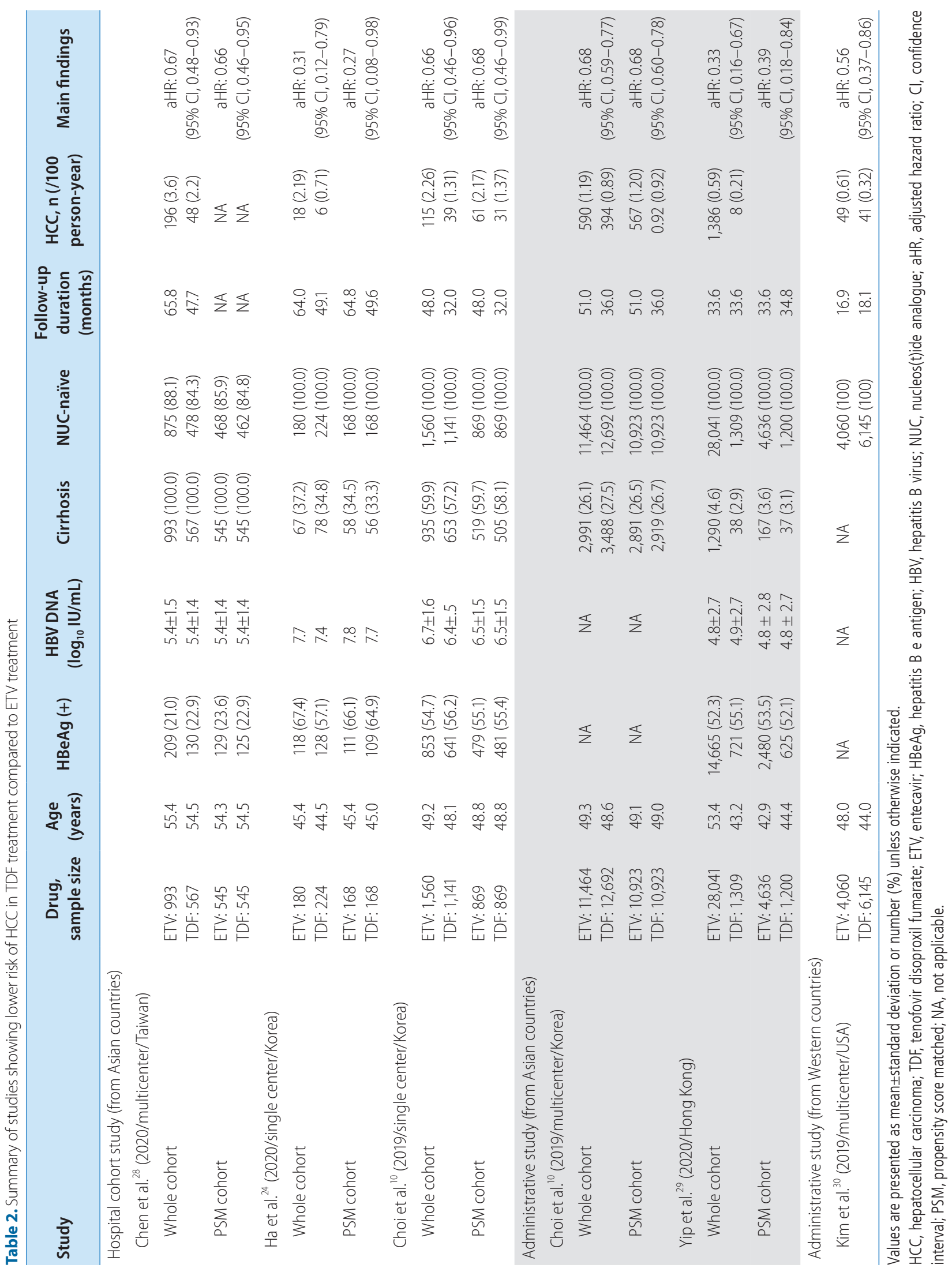




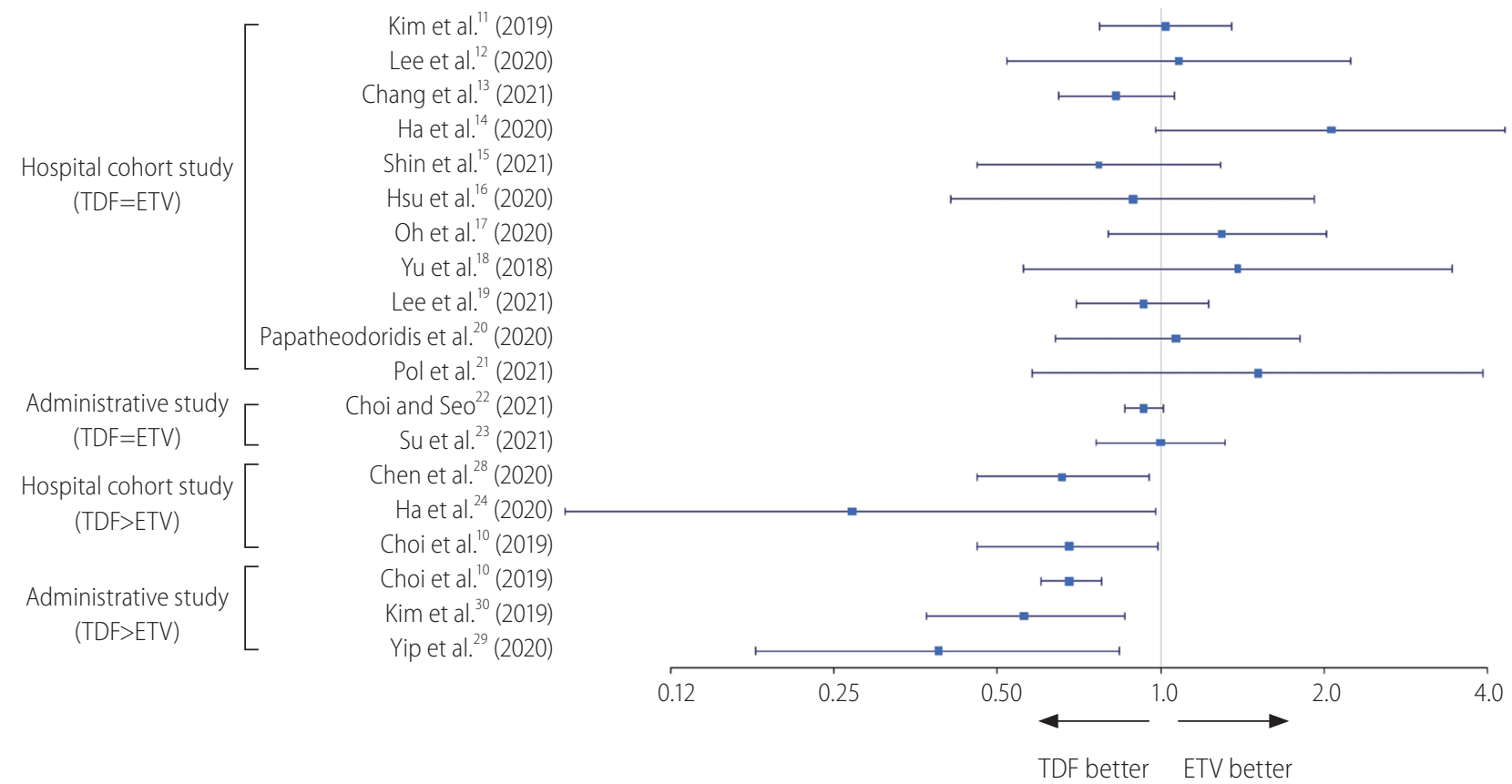

Figure 1. Forest plot of the incidence rates of hepatocellular carcinoma in studies that compared preventive effects of TDF and ETV after the matching of baseline variables. Cl, confidence interval; TDF, tenofovir disoproxil fumarate; ETV, entecavir.

\section{Western study}

A retrospective cohort study using the Corporate Data Warehouse from the Veterans Information Systems and Technology Architecture in the United States analyzed 3,287 patients with a mean follow-up period of 5.4 years. In the unadjusted analysis, a lower tendency of HCC occurrence was observed in the ETV group compared to the TDF group. However, PSM analysis showed similar risk of HCC between ETV and TDF-treated patients (aHR, 1.00; $95 \% \mathrm{Cls}, 0.76-1.32)$. Also, statistically similar risk of death or LT (aHR, 1.16; 95\% Cls, 0.98-1.39) was observed between the two treatment groups. ${ }^{23}$

\section{TENOFOVIR IS BETTER THAN ENTECAVIR FOR THE PREVENTION OF HCC}

\section{Hospital-based cohort studies}

\section{Asian studies}

A large-scale single-center study by Choi et al. ${ }^{10}$ was the first to report that TDF treatment had a significantly lower risk of HCC compared to ETV treatment (Table 2). This study demonstrated that patients treated with TDF, compared with ETV, showed 34\% and $32 \%$ reductions in their risk for HCC by multivariable and PSM analyses, respectively. ${ }^{10}$ In addition, patients treated with TDF showed significantly higher virological responses $(85.2 \%$ [TDF] vs. 78.7\% [ETV], P<0.001) and ALT normalization rates, according to the American Association for the Study of Liver Diseases 2015 criteria (44.3\% [TDF] vs. $38.7 \%$ [ETV], $P=0.002$ ) after 1 year of treatment. ${ }^{10}$

Another single-center Korean study of 404 treatment-naive patients with CHB showed that TDF treatment was associated with a lower HCC risk by multivariable analysis (aHR, 0.31; 95\% Cls, $0.12-0.79 ; P=0.014$ ) and PSM analysis (aHR, 0.27; 95\% Cls, $0.08-0.98 ; P=0.046) .{ }^{24}$ Interestingly, when the authors adjusted for sustained virological suppression in their PSM analysis, statistical significance was not reached, despite a persistent trend of lower risk with TDF treatment (aHR, 0.36; 95\% Cls, 0.12-1.14; $P=0.08$ ). Theoretically, however, it is not possible to measure sustained virological suppression at baseline. Therefore, adding this variable into PSM might not be statistically justifiable.

Choi et al. ${ }^{25}$ also studied 1,695 patients with HBV-related HCC of Barcelona Clinic Liver Cancer stage of 0 or $A$ to see whether TDF treatment has a lower risk of HCC recurrence after curative- 
Sung Won Lee, et al. Entecavir versus tenofovir for $\mathrm{HCC}$ prevention

intent liver resection compared to ETV treatment. Notably, TDF treatment was associated with significantly lower rates of HCC recurrence (aHR, 0.82; 95\% Cls, 0.68-0.98; $P=0.03$ ) and death or transplantation (aHR, $0.62 ; 95 \% \mathrm{Cls}, 0.44-0.88 ; P=0.01$ ) by multivariable analysis. These findings were consistently reproduced in 567 PSM pairs (HR, 0.77; 95\% Cls, 0.62-0.95; $P=0.02$ for HCC recurrence and $\mathrm{HR}, 0.63 ; 95 \% \mathrm{Cls}, 0.42-0.96 ; P=0.03$ for death or transplantation). Interestingly, the magnitude of risk difference for late recurrence ( $\geq 2$ years after liver resection; $H R, 0.68$ ) was more prominent than that for early recurrence $(<2$ years after liver resection; $H R, 0.79){ }^{25}$

A Chinese study of 233 patients with CHB-related compensated cirrhosis showed that TDF treatment led to significantly longer disease-free survival compared to ETV treatment after liver resection (33 months for TDF and 24 months for ETV, $P<0.001){ }^{26}$ Another study from China also reported that TDF treatment was associated with a significantly lower rate of $\mathrm{HCC}$ recurrence (aHR, $0.67 ; 95 \% \mathrm{Cls}, 0.48-0.93 ; P=0.04)$ after liver resection compared to non-TDF treatment, such as ETV. ${ }^{27}$

A multicenter retrospective study from Taiwan, which included 1,560 cirrhotic patients with CHB, reported that TDF treatment was significantly associated with a lower risk of HCC compared to ETV treatment, as shown by multivariable analysis (aHR, 0.67; 95\% Cls, 0.48-0.93; $P=0.02$ ), PSM analysis (aHR, 0.66; 95\% Cls, $0.46-0.95 ; P=0.02)$, and IPTW analysis (aHR, 0.73; 95\% Cls, $0.54-0.98 ; P=0.04) .{ }^{28}$ Of note, the significantly lower risk of HCC in the TDF group was consistently observed in the subgroup analyses of treatment-naïve patients (aHR, 0.58; 95\% Cls, 0.40-0.84; $P=0.004)$ and patients with compensated cirrhosis at baseline (aHR, 0.69; 95\% Cls, 0.48-1.00; $P=0.049$ ). ${ }^{28}$ However, comparable risk of HCC was observed between the two treatment groups after excluding 398 patients (25.5\%) who were enrolled after 2011 , to prevent artificially minimizing the follow-up duration between the two treatments $(P=0.881$ for PSM and $P=0.879$ for IPTW analysis). ${ }^{28}$

\section{Studies using administrative databases}

\section{Asian studies}

A nationwide cohort study from Korea was the first to report the lower risk of HCC with TDF treatment than with ETV treatment. ${ }^{10}$ In this study, the risk of HCC was compared among 24,156 treatment-naïve patients with $\mathrm{CHB}$, and the results showed that TDF treatment was significantly associated with a lower risk of HCC compared to ETV treatment (aHR, 0.68; 95\% Cls, 0.59-0.77;
$P<0.001) .{ }^{10}$ This lower risk of HCC in the TDF treatment group was reproduced in the PSM analysis of 10,923 pairs (aHR, 0.68; 95\% Cls, 0.60-0.78; $P<0.001)$.

Another study from Hong Kong, which used a large administrative database, subsequently showed a lower risk of HCC in the TDF group compared to the ETV group. ${ }^{29}$ In 29,350 treatment-naive patients with CHB and a median follow-up period of 3.6 years, TDF treatment was consistently associated with a lower risk of HCC compared to ETV (weighted subdistribution; HR, 0.36; 95\% Cls, 0.16-0.80; $P=0.013)$. These results were supported by various sophisticated statistical adjustments including multivariable, PSM, IPTW, and competitive risk analyses to minimize selection bias in the retrospective study. Additionally, in this study, patients who were treated with TDF (77.6\%) showed a significantly higher virological response at 1 year compared to those who were treated with ETV (69.7\%), although ALT normalization rate at 1 year was higher in the ETV group compared to the TDF group.

\section{Western study}

A study published as a collection of meeting abstracts analyzed the U.S. administrative data comparing TDF and ETV treatments in terms of the risk of $\mathrm{HCC}$ in treatment-naivve patients with $\mathrm{CHB} .{ }^{30}$ In this study, the absolute rate of HCC was lower in those treated with TDF (0.32 person year [PY]) than in those treated with ETV (0.61 PY). In addition, multivariable analysis and weighting by propensity score showed that treatment with TDF was associated with a significantly decreased risk of HCC occurrence (aHR, 0.56; 95\% Cls, 0.37-0.86). ${ }^{30}$

\section{DISCUSSION}

In the current study, we have comprehensively reviewed recent comparative studies regarding the effects of ETV and TDF on the prevention of HCC. We have classified the studies according to regions in which the studies were conducted as well as the data sources, in order to observe the potential difference in the results depending on regions or whether the data was collected from hospital cohorts or administrative databases.

The studies that showed no difference in preventive effects between ETV and TDF have suggested the following evidence for their equal effects. First, the common features of the studies that suggested TDF superiority mostly used big data from administrative databases. These database studies have an advantage of including a large number of patients. However, they may also have 
some disadvantages, such as potential unbalanced distribution of HCC risk factors, different periods of ETV and TDF onset, and additional confounders that cannot be corrected for by any sophisticated statistical method. ${ }^{31}$ For example, in the study by Yip et al., ${ }^{29}$ one-third of the HBV DNA values and $21 \%$ of the prothrombin time values, which may be important factors for the analysis of HCC development in CHB patients, were missing, and therefore, had to be imputed before the analysis was performed. In addition, out of the 29,350 patients analyzed, there was a big difference in the number of patients included in each group; 28,041 patients were included in the ETV group, while only 1,309 patients were in the TDF group. Correspondingly, only eight cases of HCC were observed in the TDF group, which may have been too small a number of events to effectively compare the HCC incidence between the two groups. In addition, the HR was 0.39 in this study, which indicates that TDF lowers HCC incidence by $61 \%$ compared to ETV. However, in the study by Nguyen et al. ${ }^{32}$ that compared the incidence of HCC between TDF and no treatment, the HR was similar at 0.34. As ETV has demonstrated its protective effect against HCC in numerous studies, it is highly unlikely for ETV treatment to have similar effects as no treatment.

Second, there was no clear evidence that ETV contains carcinogenic property. Although an increase in lung and vascular tumors were observed in the mouse experiment, the doses used in such experiments were more than 100 -fold higher compared to the approved dose for humans. Furthermore, a long-term study that included more than 12,000 patients showed no difference in HCC and non-HCC malignancies between ETV and other NUCs. ${ }^{33}$

Third, the patient warehousing phenomenon may have resulted in TDF superiority in some studies. The patient warehousing phenomenon indicates that deferring treatment as a new effective drug is known to be released soon. As ETV was approved a few years earlier than TDF in most countries, more patients with severe chronic liver disease, who had been waiting for more potent newly available antiviral agent, may have been included in the ETV group. In addition, TDF may have been avoided in the elderly as well as patients with co-morbidities, due to concerns of renal toxicity and osteoporosis. ${ }^{16}$ In this context, the pooled 5-year cumulative HCC incidence in the most recently published meta-analysis involving more than 100,000 patients showed that TDF had significantly superior preventive effects in the unmatched population while no difference was observed in the PSM population, which may indicate the patient warehousing phenomenon. ${ }^{34}$ Similarly, expansion in the indication for treatment in guidelines and the consequent changes in the reimbursement criteria for antiviral therapy over the years may have resulted in the inclusion of less severe patients in the TDF group. For instance, in Korea, the reimbursement criteria for cirrhotic patients used to require ALT higher than the upper limits of normal prior to 2015, but these have expanded to include patients with normal ALT levels since 2015. As ETV had been approved in 2007 and TDF in 2012, more patients in the TDF group may have initiated antiviral therapy after likewise expansion in reimbursement criteria and indications for treatment.

In contrast, the studies showing superior preventive effects of TDF compared to ETV have suggested the following evidence for their differences. First, TDF might have more potent antiviral efficacy. In the study by Choi et al., ${ }^{10}$ TDF treatment showed significantly higher rates of virological response and ALT normalization at 1 year of antiviral treatment in the entire cohort and PSM. A small randomized trial comparing ETV and TDF of antiviral efficacy demonstrated a higher hepatitis B surface antigen level reduction in patients treated with TDF compared to those treated with ETV. ${ }^{35}$ These results might be linked to the superior preventive effects of TDF in reducing the HCC risk, albeit the difference in antiviral efficacy may be small.

Second, a meta-regression analysis showed that the inclusion of decompensated cirrhosis was one of the most important determinants showing superiority of the preventive effect of TDF in reducing the risk of HCC. $^{36}$ In other words, studies that included decompensated cirrhosis tended to show favorable outcomes by TDF in the risk of HCC compared to ETV. Previous studies already demonstrated that long-term antiviral treatment can lead to improvement and regression of cirrhosis in patients with $\mathrm{CHB} .{ }^{37}$ Therefore, TDF may have better preventive effect in this subset of patients.

Third, a possible biological plausibility of superior preventive effects of TDF compared to ETV was suggested in previous studies, in which a higher interferon lambda-3 level was shown in CHB patients treated with TDF than in those treated with ETV. ${ }^{38}$ Also, a potent antitumor activity of the interferon lambda-3 pathway was shown in animal models of cancer, including $\mathrm{HCC}^{39,40}$

Lastly, most of the meta-analyses, ${ }^{36,41-44}$ but not all, ${ }^{34}$ in this comparison exhibited superior chemo-preventive effect by TDF treatment compared to ETV treatment. Since meta-analysis collects numerous studies of the same topic, it is able to show a real difference that was not either statistically significant or captured due to small sample size in individual studies, if the real difference still exists. 


\section{CONCLUSIONS}

Although a larger number of studies have favored a similar efficacy between ETV and TDF in reducing the risk of HCC development, controversy remains on whether TDF reduces the risk of HCC to a greater extent compared to ETV. Several recent studies from Asia, Europe, and the U.S. did not reproduce the original findings of the study by Choi et al., ${ }^{10}$ which might suggest that the current practice and guidelines for using ETV and TDF in patients with $\mathrm{CHB}$ should not be changed. However, as all of these studies were either neutral or in favor of TDF, further studies are required to identify the subset of patient population who will benefit from TDF, rather than ETV.

\section{Authors' contributions}

SWL, JC, SUK, and YSL were involved in study concept and design; SWL, JC, SUK, and YSL were involved in critical revision of the manuscript; SWL, JC, and SUK were involved in acquisition of data and drafting of the manuscript. All authors have read and approved the manuscript.

\section{Conflicts of Interest}

The authors have no conflicts to disclose.

\section{REFERENCES}

1. Kim BK, Revill PA, Ahn SH. HBV genotypes: relevance to natural history, pathogenesis and treatment of chronic hepatitis B. Antivir Ther 2011;16:1169-1186.

2. Chen CJ, Yang HI, Su J, Jen CL, You SL, Lu SN, et al. Risk of hepatocellular carcinoma across a biological gradient of serum hepatitis $B$ virus DNA level. JAMA 2006;295:65-73.

3. Korean Association for the Study of the Liver (KASL). KASL clinical practice guidelines for management of chronic hepatitis B. Clin Mol Hepatol 2019;25:93-159.

4. Wong GL, Chan HL, Mak CW, Lee SK, Ip ZM, Lam AT, et al. Entecavir treatment reduces hepatic events and deaths in chronic hepatitis B patients with liver cirrhosis. Hepatology 2013;58:1537-1547.

5. Song DS, Kim W, Ahn SH, Yim HJ, Jang JY, Kweon YO, et al. Continuing besifovir dipivoxil maleate versus switching from tenofovir disoproxil fumarate for treatment of chronic hepatitis B: results of 192-week phase 3 trial. Clin Mol Hepatol 2021;27:346-359.

6. European Association for the Study of the Liver. EASL 2017 clinical practice guidelines on the management of hepatitis B virus infection. J Hepatol 2017;67:370-398.
7. Terrault NA, Lok ASF, McMahon BJ, Chang KM, Hwang JP, Jonas $M M$, et al. Update on prevention, diagnosis, and treatment of chronic hepatitis B: AASLD 2018 hepatitis B guidance. Hepatology 2018:67:1560-1599.

8. Papatheodoridis GV, Idilman R, Dalekos GN, Buti M, Chi H, van Boemmel $F$, et al. The risk of hepatocellular carcinoma decreases after the first 5 years of entecavir or tenofovir in Caucasians with chronic hepatitis B. Hepatology 2017;66:1444-1453.

9. Kim BG, Park NH, Lee SB, Lee H, Lee BU, Park JH, et al. Mortality, liver transplantation and hepatic complications in patients with treatment-naïve chronic hepatitis B treated with entecavir vs tenofovir. J Viral Hepat 2018;25:1565-1575.

10. Choi J, Kim HJ, Lee J, Cho S, Ko MJ, Lim YS. Risk of hepatocellular carcinoma in patients treated with entecavir vs tenofovir for chronic hepatitis B: a Korean nationwide cohort study. JAMA Oncol 2019;5:30-36.

11. Kim SU, Seo YS, Lee HA, Kim MN, Lee YR, Lee HW, et al. A multicenter study of entecavir vs. tenofovir on prognosis of treatmentnaïve chronic hepatitis B in South Korea. J Hepatol 2019;71:456-464.

12. Lee SW, Kwon JH, Lee HL, Yoo SH, Nam HC, Sung PS, et al. Comparison of tenofovir and entecavir on the risk of hepatocellular carcinoma and mortality in treatment-naïve patients with chronic hepatitis B in Korea: a large-scale, propensity score analysis. Gut 2020;69:1301-1308.

13. Chang TS, Yang YH, Chen WM, Shen CH, Tung SY, Yen CW, et al. Long-term risk of primary liver cancers in entecavir versus tenofovir treatment for chronic hepatitis B. Sci Rep 2021;11:1365.

14. Ha I, Chung JW, Jang ES, Jeong SH, Kim JW. Comparison of the ontreatment risks for hepatocellular carcinoma between entecavir and tenofovir: a propensity score matching analysis. J Gastroenterol Hepatol 2020;35:1774-1781.

15. Shin JW, Jeong J, Jung SW, Lee SB, Park BR, Kim MJ, et al. Comparable incidence of hepatocellular carcinoma in chronic hepatitis B patients treated with entecavir or tenofovir. Dig Dis Sci 2021;66:1739 1750.

16. Hsu YC, Wong GL, Chen CH, Peng CY, Yeh ML, Cheung KS, et al. Tenofovir versus entecavir for hepatocellular carcinoma prevention in an international consortium of chronic hepatitis B. Am J Gastroenterol 2020;115:271-280.

17. Oh H, Yoon EL, Jun DW, Ahn SB, Lee HY, Jeong JY, et al. No difference in incidence of hepatocellular carcinoma in patients with chronic hepatitis b virus infection treated with entecavir vs tenofovir. Clin Gastroenterol Hepatol 2020;18:2793-2802.e6.

18. Yu JH, Jin YJ, Lee JW, Lee DH. Remaining hepatocellular carcinoma risk in chronic hepatitis B patients receiving entecavir/tenofovir in South Korea. Hepatol Res 2018;48:862-871.

19. Lee JH, Kim BK, Park SY, Tak WY, Park JY, Kim DY, et al. The efficacies of entecavir and tenofovir in terms of enhancing prognosis 
after curative treatment of hepatitis B virus-related hepatocelIular carcinoma. Eur J Intern Med. 2021 Mar 30. doi: 10.1016/ j.ejim.2021.02.019.

20. Papatheodoridis GV, Dalekos GN, Idilman R, Sypsa V, Van Boemmel $F$, Buti $M$, et al. Similar risk of hepatocellular carcinoma during long-term entecavir or tenofovir therapy in Caucasian patients with chronic hepatitis B. J Hepatol 2020;73:1037-1045.

21. Pol S; ANRS/AFEF study group. Similar 5-year HCC occurrence in tenofovir- and entecavir-treated HBV chronic infection in the French AFEF/ANRS CO22 hepather cohort. Aliment Pharmacol Ther 2021;53:616-629.

22. Choi H, Seo GH. Entecavir versus tenofovir for the prevention of hepatocellular carcinoma in treatment-naïve chronic hepatitis B patients in Korea. J Korean Med Sci 2021;36:e89.

23. Su F, Berry K, Ioannou GN. No difference in hepatocellular carcinoma risk between chronic hepatitis $B$ patients treated with entecavir versus tenofovir. Gut 2021;70:370-378.

24. Ha Y, Chon YE, Kim MN, Lee JH, Hwang SG. Hepatocellular carcinoma and death and transplantation in chronic hepatitis $B$ treated with entecavir or tenofovir disoproxil fumarate. Sci Rep 2020;10:13537.

25. Choi J, Jo C, Lim YS. Tenofovir versus Entecavir on recurrence of hepatitis B virus-related hepatocellular carcinoma after surgical resection. Hepatology 2021;73:661-673.

26. Zhang M, Wang D, Liu H, Li H. Tenofovir decrease hepatocellular carcinoma recurrence in chronic hepatitis B patients after liver resection. Infect Agent Cancer 2018;13:19.

27. Ge Z, Ma J, Qiao B, Wang Y, Zhang H, Gou W. Impact of tenofovir antiviral treatment on survival of chronic hepatitis $B$ related hepatocellular carcinoma after hepatectomy in Chinese individuals from Qingdao municipality. Medicine (Baltimore) 2020;99:e21454.

28. Chen $\mathrm{CH}$, Chen $\mathrm{CY}$, Wang JH, Lai HC, Hung CH, Lu SN, et al. Comparison of incidence of hepatocellular carcinoma between chronic hepatitis B patients with cirrhosis treated with entecavir or tenofovir in Taiwan - a retrospective study. Am J Cancer Res 2020;10:38823895.

29. Yip TC, Wong VW, Chan HL, Tse YK, Lui GC, Wong GL. Tenofovir is associated with lower risk of hepatocellular carcinoma than entecavir in patients with chronic HBV infection in China. Gastroenterology 2020;158:215-225.e6.

30. Kim WR, Telep L, Lu M, Ramroth H, Flaherty JF, Gaggar A, et al. Risk of incident hepatocellular carcinoma in hepatitis B-infected patients treated with tenofovir disoproxil fumarate versus entecavir: a US administrative claims analysis. Hepatology 2019;70:302A-303A.

31. Lampertico P, Papatheodoridis GV. Is tenofovir superior to entecavir in reducing the risk of hepatocellular carcinoma in chronic hepatitis $B$ ? The controversy continues. Gastroenterology 2020;158:42-44.

32. Nguyen MH, Yang HI, Le A, Henry L, Nguyen N, Lee MH, et al. Reduced incidence of hepatocellular carcinoma in cirrhotic and non- cirrhotic patients with chronic hepatitis B treated with tenofovir-A propensity score-matched study. J Infect Dis 2019;219:10-18.

33. Hou JL, Zhao W, Lee C, Hann HW, Peng CY, Tanwandee T, et al. Outcomes of long-term treatment of chronic HBV infection with entecavir or other agents from a randomized trial in 24 countries. Clin Gastroenterol Hepatol 2020;18:457-467.e21.

34. Tseng CH, Hsu YC, Chen TH, Ji F, Chen IS, Tsai YN, et al. Hepatocellular carcinoma incidence with tenofovir versus entecavir in chronic hepatitis B: a systematic review and meta-analysis. Lancet Gastroenterol Hepatol 2020;5:1039-1052.

35. Koike K, Suyama K, Ito H, Itoh H, Sugiura W. Randomized prospective study showing the non-inferiority of tenofovir to entecavir in treatment-naïve chronic hepatitis B patients. Hepatol Res 2018;48: 59-68.

36. Choi WM, Choi J, Lim YS. Effects of tenofovir vs entecavir on risk of hepatocellular carcinoma in patients with chronic HBV infection: a systematic review and meta-analysis. Clin Gastroenterol Hepatol 2021;19:246-258.e9.

37. Marcellin P, Gane E, Buti M, Afdhal N, Sievert W, Jacobson IM, et al. Regression of cirrhosis during treatment with tenofovir disoproxil fumarate for chronic hepatitis B: a 5-year open-label follow-up study. Lancet 2013;381:468-475.

38. Murata K, Asano M, Matsumoto A, Sugiyama M, Nishida N, Tanaka $\mathrm{E}$, et al. Induction of IFN-lambda3 as an additional effect of nucleotide, not nucleoside, analogues: a new potential target for HBV infection. Gut 2018;67:362-371.

39. Sato A, Ohtsuki M, Hata M, Kobayashi E, Murakami T. Antitumor activity of IFN-lambda in murine tumor models. J Immunol 2006;176:7686-7694.

40. Abushahba W, Balan M, Castaneda I, Yuan Y, Reuhl K, Raveche

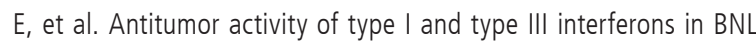
hepatoma model. Cancer Immunol Immunother 2010;59:1059-1071.

41. Dave S, Park S, Murad MH, Barnard A, Prokop L, Adams LA, et al. Comparative effectiveness of entecavir versus tenofovir for preventing hepatocellular carcinoma in patients with chronic hepatitis B: a systematic review and meta-analysis. Hepatology 2021;73:68-78.

42. Li M, Lv T, Wu S, Wei W, Wu X, Ou X, et al. Tenofovir versus entecavir in lowering the risk of hepatocellular carcinoma development in patients with chronic hepatitis B: a critical systematic review and meta-analysis. Hepatol Int 2020;14:105-114.

43. Gu L, Yao Q, Shen Z, He Y, Ng DM, Yang T, et al. Comparison of tenofovir versus entecavir on reducing incidence of hepatocellular carcinoma in chronic hepatitis B patients: a systematic review and meta-analysis. J Gastroenterol Hepatol 2020;35:1467-1476.

44. Liu H, Shi Y, Hayden JC, Ryan PM, Rahmani J, Yu G. Tenofovir treatment has lower risk of hepatocellular carcinoma than entecavir treatment in patients with chronic hepatitis B: a systematic review and meta-analysis. Liver Cancer 2020;9:468-476. 